\title{
Application of Nanoparticles in the Process of Cancer Diagnosis and Therapy
}

\author{
Shiyao Sun", Hao Zhang", Yiyang Li", Mi Zhang, Mengyao Zhang, Yantao Han* and Wenwen \\ Zhao* \\ School of Basic Medical Sciences, Qingdao University, China \\ \#These authors contributed equally to this work \\ *Corresponding author: Wenwen Zhao, Qingdao University Medical College, 308 Ningxia Road, Qingdao, Shandong, 266021, China. \\ Han yantao, Qingdao University Medical College, 308 Ningxia Road, Qingdao, Shandong, 266021, China. \\ To Cite This Article: Mengyao Zhang, Wenwen Zhao, Application of Nanoparticles in the Process of Cancer Diagnosis and Therapy. Am J Biomed \\ Sci \& Res. 2021 - 12(1). AJBSR.MS.ID.001704. DOI: 10.34297/AJBSR.2021.12.001704.
}

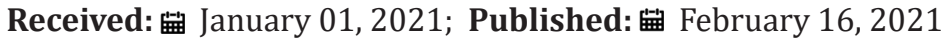

\begin{abstract}
As the incidence of malignant tumors is increasing globally, fighting cancer has become an urgently international issue. Considering that traditional therapy as chemotherapy and radiotherapy explored a series of disadvantages in clinic including killing normal cells and reducing immunity, a series of new strategies and means are emerged to treat cancers preferably. Among, nanoparticles exhibit their noticeably advantages on both cancer early detection and future treatment. In this review, we do an intensive research on nanotechnology-based treatments for cancer.

Keywords: Cancer, Nanoparticles, Drug delivery, Thermal therapy
\end{abstract}

\section{Introduction}

All along, cancer is considered as an extremely grave threat to public health. According to the International Center for Research on Cancer, approximately 9 million people worldwide die of malignant tumor every year [1]. Generally, accurate and effective early detection for cancer will offer patients optimal treatment time. At present, poor pharmacokinetic and serious damages on human body tremendously limited contrast agents 'clinical application for early diagnosis. Nanotechnology, a material with remarkably varied and new properties, provides new molecular contrast agents and materials to enable earlier and more accurate initial diagnosis [2]. In addition, although many chemotherapy drugs for cancer treatment are available, their potential toxicity is the main point of concern. The good news is that nanoparticles offer enormous potential in creating targeted and highly effective anti-cancer treatment [3]. Exactly speaking, nanoparticles, as drug carriers, increase the targeting of chemotherapeutic drugs [4]. In this review, the progress of nanoparticle research in cancer diagnosis and treatment is reviewed in detail, and the prospect and challenges of its clinical application are prospected.

\section{Nanoparticles and Cancer Diagnosis}

Contrast agents including gadolinium and sodium iodide are vital chemicals that used for CT and fluoroscopic imagining which are widely applied for disease diagnosis, especially for cancer early detection [5,6]. Nowadays, contrast agents' poor pharmacokinetic properties and low sensitivity hinder and limit their further development around cancer imaging. For example, the first generation of Gd-based contrast agents which are currently used in magnetic resonance imaging (MRI) have been reported to cause nephrotoxicity [7]. Here, the advent of nanotechnology has revolutionized the traditional contrast agents. Magnetic nanoparticles are combined with specific molecular markers which were remarkably expressed on the surface of tumor cells [8]. Obviously, reformed nanoparticles could specifically identify tumors subsequently form magnetic resonance images. This means can not only reduce the amount of contrast agent, but also increase the sensitivity of the imaging. In addition, superpara magneticiron oxidenano particles (SPIONs) [9], another important type of nanoparticles, are also modified with specific ligands to 
identify exclusive tissues and organs. The bioavailability of drugs in tumor sites can be improved by double-effect targeting with high permeability and external magnetic field, to reduce toxicity to normal cells [10]. Nanoparticles, as a new generation contrast agent, must have a broad application prospect in cancer diagnosis.

\section{Nanoparticles and Cancer Targeting Therapy}

\section{Nanoparticles and Drug Delivery}

Despite the lack of specificity and systemic toxicity, chemotherapy is still the most commonly used treatment for cancer. [11]. Using nanoparticles to carry chemotherapeutic drugs to target tumors is a good way to avoid untargeted therapy with chemotherapeutic agents. For example, magnetic nanoparticle, one important member of the nanoparticle family, gathers to the target site under the action of magnetic field, and releases antitumor drugs in the site at a certain rate of drug release. Also, $\mathrm{Wu}$ et al. encapsulated superparamagnetic iron oxide particles with polydopamine to obtain magnetic nanoparticles with an average particle size of $80 \mathrm{~nm}$. The external magnetic field significantly promoted the killing effect of polydopamine on HeLa cells, which proved that the external magnetic field had a significant effect on the aggregation of magnetic nanoparticles [12]. Besides, Munaweer et al. proved that magnetic nanoparticles containing platinum chemotherapeutic drugs can be used as effective chemotherapeutic and radiotherapy drugs. The surface engineering graphene oxide nanoparticles (GO) can be prepared by covalently linking with amine-functionalized iron oxide nanoparticles (IONP). IONP can be directed to specific organs, tissues or tumors through external magnetic fields. GO is non-toxic to normal cells but can have toxic effects on tumor cells [13].

\section{Nanoparticles and Thermal Therapy}

The rapid development of nanoparticles has led to a new technique for treating cancer called hyperthermia [14]. The technique is focal and repeatable with a minimally invasive application [15]. First, nanoparticles target to bind to tumors. The nanoparticles are then heated by an external technique to produce heat that kills the tumor. Photothermal therapy, for example, uses lasers to heat nanoparticles, selectively killing the cells that contain them. Chen et al. reported that 4T-1 cancer cell activity was greatly inhibited after nanoparticles treatment and near-infrared radiation of Polyethylene glycol (peg) coated nanoparticles [16]. However, photothermal therapy is still a challenge in treating tumors due to the low light penetration of tissues. Superparamagnetic AuNPs cluster showed typical superparamagnetic behavior and supernormal magnetic hyperthermia effect, which was more beneficial to cancer treatment than photothermal treatment alone [17]. It has also been reported that photo hyperthermia combined with magnetic hyperthermia for greater effect. In the case of multifunctional silver/magnetite nanophere (Ag/Fe304), the presence of an external magnetic field and simultaneous laser irradiation produce more heat to kill cancer cells [9].

\section{Conclusion}

In our study, nanotechnology significantly increases the bioavailability of insoluble drugs and improves drug release and absorption behaviour. It improves the targeting ability of drugs to tumor tissue sites and increases drug distribution and accumulation. All the changes can reduce drug toxicity and side effects to normal tissues and cells and reduce the toxicity and efficacy enhancement. The current condition is that although nanotechnology is widely studied, it is not yet ready to be applied to the clinical treatment for cancer. Several issues that need to be addressed such as whether the particle size and surface properties of nanoparticles meet regulatory requirements and so on. Also, the emergence of more complex nanoparticle systems requires better methods for evaluating the biocompatibility of cells.

Overall, there is still a long way to go for nanoparticles to be clinically viable, but their potential advantages will drive them towards success.

\section{Conflict of Interest}

The authors declare that the research was conducted in the absence of any commercial or financial relationships that could be construed as a potential conflict of interest.

\section{References}

1. Ferlay J, Colombet M, Soerjomataram I, Mathers C, Parkin DM, et al. (2019) Estimating the global cancer incidence and mortality in 2018: GLOBOCAN sources and methods. Int J cancer 144(8): 1941-1953.

2. Suárez García S, Arias Ramos N, Frias C, Candiota, Arús C, et al. (2018) Dual T/ T Nanoscale Coordination Polymers as Novel Contrast Agents for MRI: A Preclinical Study for Brain Tumor. ACS Appl Mater Interfaces 10(45): 38819-38832.

3. Petros RA, DeSimone JM (2010) Strategies in the design of nanoparticles for therapeutic applications. Nature reviews. Nat Rev Drug Discov 9(8): 615-627.

4. Hassan S, Prakash G, Ozturk A, Saghazadeh S, Sohail M, et al. (2017) Evolution and Clinical Translation of Drug Delivery Nanomaterials. Nano Today 15: 91-106

5. Wyskocka Gajda M, Przypis Ł, Olesiejuk M, Krawczyk T, Kuźnik A, et al. (2020) A step towards gadolinium-free bioresponsive MRI contrast agent. Eur J Med Chem 211: 113086.

6. Fruhwirth G, Diocou S, Blower P, Ng T, Mullen G (2014) A whole-body dual-modality radionuclide optical strategy for preclinical imaging of metastasis and heterogeneous treatment response in different microenvironments. J Nucl Med 55(4): 686-694.

7. Shen Z, Fan W, Yang Z, Liu Y, Bregadze V, et al. (2019) Exceedingly Small Gadolinium Oxide Nanoparticles with Remarkable Relaxivities for Magnetic Resonance Imaging of Tumors. Small 15(41): e1903422.

8. KneŽević N, Gadjanski I, Durand (2019) Magnetic nanoarchitectures for cancer sensing, imaging, and therapy. J Mater Chem B 7(1): 9-23. 
9. Gan S, Lin Y, Feng Y, Shui L, Li H, et al. (2018) Magnetic polymeric nanoassemblies for magnetic resonance imaging-combined cancer theranostics. Int J Nanomedicine 13: 4263-4281.

10. Tay Z, Chandrasekharan P, Chiu Lam A, Hensley D, Dhavalikar R, et al. (2018) Magnetic Particle Imaging-Guided Heating in Vivo Using Gradient Fields for Arbitrary Localization of Magnetic Hyperthermia Therapy. ACS Nano 12(4): 3699-3713.

11. Peer D, Karp J, Hong S, Farokhzad O, Margalit R, et al. (2007) Nanocarriers as an emerging platform for cancer therapy. Nat Nanotechnol 2(12): 751-760.

12. Wu M, Zhang D, Zeng Y, Wu L, Liu X, et al. (2015) Nanocluster of superparamagnetic iron oxide nanoparticles coated with poly (dopamine) for magnetic field-targeting, highly sensitive MRI and photothermal cancer therapy. Nanotechnology 26(11): 115102.
13. Munaweera I, Shi Y, Koneru B, Saez R, Aliev A, et al. (2015) Chemoradiotherapeutic Magnetic Nanoparticles for Targeted Treatment of Nonsmall Cell Lung Cancer. Mol Pharm 12(10): 3588-3596.

14. Chang M, Hou Z, Wang M, Li C, Lin J (2020) Recent Advances in Hyperthermia Therapy-Based Synergistic Immunotherapy. Adv Mater e2004788.

15. Ding B, Zheng P, Ma P, Lin J (2020) Manganese Oxide Nanomaterials: Synthesis, Properties, and Theranostic Applications. Adv Mater 32(10): e1905823.

16. Das P, Fatehbasharzad P, Colombo M, Fiandra L, Prosperi D (2019) Multifunctional Magnetic Gold Nanomaterials for Cancer. Trends Biotechnol 37(9): 995-1010.

17. Goddard Z, Marín M, Russell D, Searcey M (2020) Active targeting of gold nanoparticles as cancer therapeutics. Chem Soc Rev 49(23): 8774-8789. 